\title{
ENTRY VEHICLE CONTROL SYSTEM DESIGN FOR THE MARS SMART LANDER
}

\author{
Philip C. Calhoun * and Eric M. Queen * \\ NASA Langley Research Center, Hampton, VA 23681
}

\begin{abstract}
The NASA Langley Research Center, in cooperation with the Jet Propulsion Laboratory, participated in a preliminary design study of the Entry, Descent and Landing phase for the Mars Smart Lander Project. This concept utilizes advances in Guidance, Navigation and Control technology to significantly reduce uncertainty in the vehicle landed location on the Mars surface. A candidate entry vehicle controller based on the Reaction Control System controller for the Apollo Lunar Excursion Module digital autopilot is proposed for use in the entry vehicle attitude control. A slight modification to the phase plane controller is used to reduce jet-firing chattering while maintaining good control response for the Martian entry probe application. The controller performance is demonstrated in a six-degree-of-freedom simulation with representative aerodynamics.
\end{abstract}

\section{Nomenclature}

$\begin{array}{ll}C G & \text { - Center-of-Gravity } \\ L E M & \text { - Lunar Excursion Module } \\ M S L & \text { - Mars Smart Lander } \\ P O S T & \text { - Program to Optimize Simulated Trajectories } \\ R C S & \text { - Reaction Control System } \\ 6 D O F & \text { - Six-Degrees-of-Freedom } \\ \alpha_{R C S} & \text { - Angular acceleration from RCS } \\ \theta_{D B} & \text { - Angle deadband } \\ \theta_{e} & \text { - Angle error }\end{array}$

\section{$\underline{\text { Introduction }}$}

The NASA Langley Research Center, in cooperation with the Jet Propulsion Laboratory, participated in a preliminary design study of the Entry, Descent and

\footnotetext{
* Aerospace Engineer, Vehicle Analysis Branch, Aerospace Systems, Concepts, and Analysis Competency

Copyright $\subseteq 2002$ by the American Institute of Aeronautics and Astronautics, Inc. No copyright is asserted in the United States under Title 17, U.S. Code. The U.S. Government has a royalty-free license to exercise all rights under the copyright claimed herein for Governmental Purposes. All other rights are reserved by the copyright owner.
}

Landing phase for the Mars Smart Lander (MSL) Project. This concept utilizes advances in Guidance, Navigation and Control technology to significantly reduce uncertainty in the vehicle landed location on the Mars surface, allowing scientific missions to specific surface features. This paper documents a candidate entry vehicle controller design for the Smart Lander project. The entry vehicle has a conical fore-body and a truncated bi-conical aft-body as shown in Figure 1. The entry phase of flight begins at the atmospheric interface and ends at the supersonic parachute deployment at about Mach 2.0. A second control system is required for the powered descent phase, after the lander is released from the subsonic parachute. The powered descent control system is not addressed in this paper.

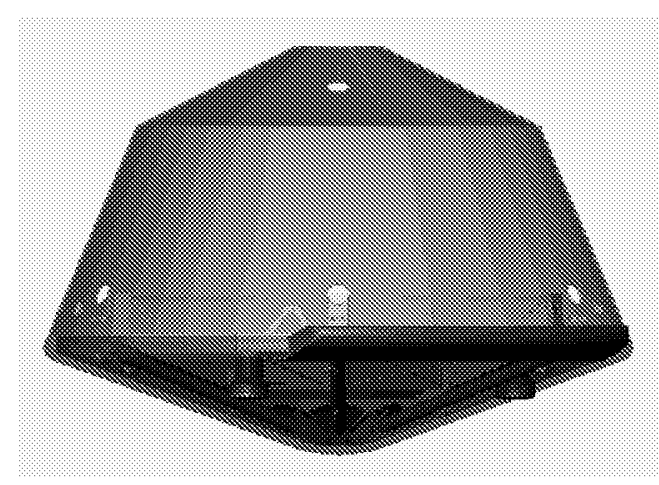

Figure 1 - Typical Entry Vehicle Configuration

During atmospheric entry the vehicle is flown with its axis of symmetry at an angle to the relative velocity direction (angle-of-attack). The resulting airflow over the vehicle creates the lift, which is used by the guidance system to reach the desired landing site. Orientation of the lift vector, via bank angle steering, allows the guidance system to maneuver the vehicle to the parachute deployment flight conditions (i.e., Mach number, dynamic pressure, and altitude). The controller objective is to maintain the entry vehicle angle-ofattack, bank angle and sideslip-angle, within specified tolerances of the commanded values. The angle-ofattack is commanded to follow the predicted pitch trim values across the Mach range. To accomplish coordinated bank maneuvers the sideslip angle is commanded to zero during the entry phase. Control actuation is by 
eight Reaction Control System (RCS) thrusters mounted in pairs at four locations as shown in figure 2 .

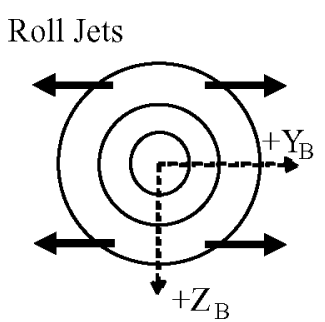

(Probe Aft View)

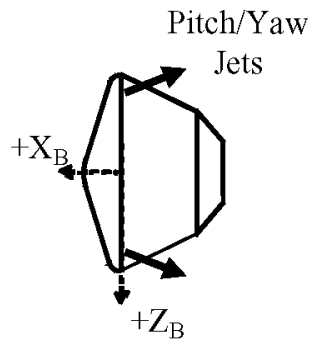

(Probe Side View)
Figure 2-RCS Thruster Layout

Two methods were considered to maintain pitch torque equilibrium at these attitudes (1) a radial Center-of-Gravity (CG) offset, and (2) an aerodynamic trim surface, either fixed or deployed. An aerodynamic trim surface is shown in the representative configuration, Figure 1, protruding out along the maximum diameter of the aeroshell forebody. The CG offset configuration was chosen as the project baseline and the controller design was developed using this assumption. To be consistent with the coordinate system conventions shown in Figure 2 the radial CG offset would be located along the $-Z_{B}$ coordinate direction. The CG offset configuration has no significant roll stiffness or damping and therefore the lateral and directional axes are aerodynamically de-coupled. This allows for a single-axis control design methodology for the pitch, yaw, and roll axes separately. The phase plane controller is well suited to control systems in which the actuators are on/off devices and there is little interaction between the axes. Therefore, a phase plane controller for each axis was chosen as the candidate approach. ${ }^{1}$ The aero tab configuration is assumed to have minimal roll stiffness and hence the single-axis controller design methodology developed in this paper is considered to be applicable in that case.

During the entry phase bank reversals are used to adjust the vehicle trajectory to meet specific flight conditions for the supersonic parachute deploy at about Mach 2.0. Reversals of $180 \mathrm{deg}$ or more may be commanded to adjust the nominal trajectory downrange and crossrange. The flight mechanics team, using the Program for the Optimization of Simulated Trajectories (POST) analysis tool, provided flight conditions for a typical entry. ${ }^{2}$ Nominal values of angle-of-attack and dynamic pressure for a typical entry trajectory are shown in Figure 3.
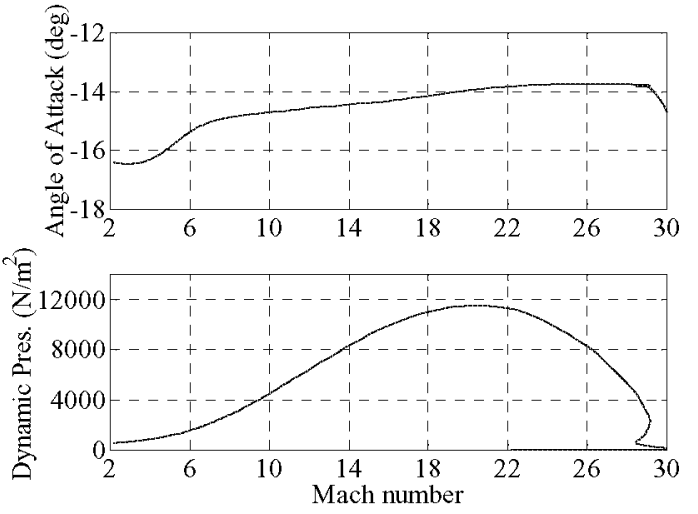

Figure 3 - Nominal Entry Trajectory

The entry flight control system will utilize RCS jets to control and stabilize the vehicle attitude during the entry phase of flight. The controller design is based on the RCS controller from the Lunar Excursion Module (LEM) digital autopilot. ${ }^{3,4}$ The LEM RCS controller used a phase plane technique that provided a trade between minimizing maneuver time and fuel usage. ${ }^{3}$ The phase plane is defined as a plot of rate error versus position error. The control approach is based on selection of on/off RCS firing commands, determined by location of the states relative to "switching curves" in the phase plane.

Because the RCS system has a finite minimum impulse, the system will not, in general, be able to reach the commanded state exactly. One of the challenges of developing a controller for this type of system is to bring the terminal condition close to the commanded condition with minimal overshoot during large angle maneuvers. Conversely, when the error is large, the control system should respond with full control effort. The guidance system requires a minimum bank acceleration of about $5 \mathrm{deg} / \mathrm{s}^{2}$ to achieve the desired targeting capability. This requirement drives the thruster size of the RCS, depending also upon the moments of inertia of the vehicle.

Performance of the candidate RCS control law was analyzed using a non-linear 6DOF simulation of flight conditions. The results include dynamic responses to representative guidance commands and estimates of RCS firings and fuel usage. The 6DOF simulation includes the Mars Global Reference Atmospheric Model $2000^{5}$, as well as a simplified gravity model including the $\mathrm{J} 2$ perturbation from a spherical field. This simulation was written using the SIMULINK ${ }^{\mathrm{TM}} 6$ toolbox in MATLAB $^{\text {TM. }}{ }^{7}$ 


\section{Model Descriptions}

\section{Aerodynamics}

The entry vehicle aerodynamic database was developed at Langley Research Center. ${ }^{8}$ It is based on Computational Fluid Dynamics calculations and measurements taken in the wind tunnel. The database includes 6DOF force and moment coefficients from noncontinuum flow conditions through hypersonic flight down to Mach 1.4 with total angle-of-attack ranging from 0 to $16 \mathrm{deg}$. The database was extrapolated at low supersonic Mach numbers for angle-of-attack up to 19 deg. This database was used for the purposes of evaluating the Smart Lander controller design in a $6 \mathrm{DOF}$ simulation.

\section{$\underline{\text { RCS Configuration }}$}

The RCS jets used for entry control are located on the backshell near the maximum diameter as shown in Figure 2. Four nearly tangential jets are used to provide roll torques for bank angle maneuvers. Four aft-firing jets, each coincidently located with a roll jet, provide pitch/yaw torques to control the vehicle angle-of-attack and to maintain zero sideslip for coordinated bank maneuvers. This configuration was chosen to provide roll control independent from yaw control, allowing adjustment of the roll/yaw control acceleration ratio over a range of angle-of-attack and vehicle inertia values. This is necessary to maintain zero sideslip during bank maneuvers. Because of the inherent stability in the pitch channel, and the small commands anticipated, independent control of pitch and yaw was considered unnecessary. For the purpose of this initial controller evaluation, the RCS jets were modeled as perfect on/off devices with an instantaneous rise time, no internal lags, and no thrust decay. RCS commands are updated at a $50 \mathrm{~Hz}$ rate, which is assumed to be consistent with the minimum on time for the thrusters.

Because pitch and yaw are controlled with four aftfiring thrusters, they cannot be commanded independently. That is, if a command is given for pitching moment and yawing moment simultaneously, the thrusters cannot provide both at their full capability. Simultaneous pitch/yaw actuation results in jet firing oncommands of diagonally offset thrusters. As can be seen from the RCS configuration in Figure 2 this would produce equal and opposite moments about the same axis resulting in zero net moment and a waste of fuel. Whenever this happens, the diagonally opposite pair of jet firing commands is ignored and only the remaining jet firing is executed in the RCS. This results in half control effort for both pitch and yaw during this command period. Since the vehicle has good static pitch stability across the Mach range, few pitch axis firings were noted in simulations studies. Vehicle closed loop performance was not degraded noticeably during infrequent simultaneous pitch/yaw commands. Since the desired roll and yaw motions are tightly coupled during large bank maneuvers, independent control of the roll and yaw channels is considered more important than tight control of the pitch channel. With this in mind, alternate jet selection logic could be set to give the yaw channel priority over the pitch channel during simultaneous commands. However, this approach was not implemented since the baseline approach demonstrated good performance in the simulation studies.

\section{Phase Plane Controller}

A phase-plane controller is based on behavior of the vehicle dynamics in the plane defined by state errors vs. state rate errors. For instance, sideslip error vs sideslip rate error would define a phase plane in the directional channel of the vehicle. Experience has shown that the Mars Smart Lander (MSL) entry attitude dynamics can be treated as a set of decoupled singleinput, single output systems as long as the pitch and yaw angular rates are kept small with moderate roll rates. The single axis phase plane controller approach performed well in the $6 \mathrm{DOF}$ simulation with roll rates as high as $20 \mathrm{deg} / \mathrm{sec}$ during large angle maneuvers. The design of a phase plane controller involves selection of switching curves that define regions within the phase plane of on/off commands for each RCS channel. These switching curves should be selected to drive the state error trajectory to the desired limit cycle, i.e. to a within a specified angle error deadband. Large angle maneuvers should be accomplished quickly with minimal command overshoot.

The phase-plane controller methodology was chosen for use in the RCS control law for the Apollo Lunar Excursion Module (LEM) digital autopilot system. ${ }^{3,4}$ For the LEM mission, there were no aerodynamic forces or moments, so the only coupling between channels was due to inertial coupling. As long as the rotation rates were sma1l, this coupling was not significant, and each axis (roll, pitch, yaw) could be treated as an independent double integrator. The resulting trajectory within the phase plane is parabolic during constant jeton firing conditions. Thus, an appropriately defined parabolic curve, which terminates at a location within the phase plane near the origin, would define a set of suitable state conditions for switching the RCS jets on. ${ }^{3,4}$ This curve defines a boundary within the phase

3

American Institute of Aeronautics and Astronautics 
plane separating regions of jet on firing commands from jet off commands. These regions are called firing and coast regions respectively. The curvature is determined by the thruster angular acceleration, $\alpha_{R C S}$, with terminus at a location of zero rate error and angle error of $+/-\theta_{D B}$. Values of the angle, $\theta_{e}$, and rate errors, $\dot{\theta}_{e}$, that are solutions of Equation (1), for both positive and negative values of $\alpha_{R C S}$, define trajectories in the phase plane that bound regions of jet on/off conditions. ${ }^{3,4}$

$$
\theta_{e}= \pm \theta_{D B}+\frac{1}{2 \alpha_{R C S}} \dot{\theta}_{e}^{2}
$$

Consider a typical roll channel response with initial angle error of $-10 \mathrm{deg}$ and a roll command of zero to demonstrate the behavior of the LEM type phase plane controller. The phase plane plot with appropriately defined parabolic switching curves terminating at an angle deadband specification of $+/-2$ deg is shown in Figure 4. The roll error is defined as roll minus roll command and the roll rate error is defined similarly. Figure 5 shows the corresponding time domain response and the associated roll torque from the RCS firings. In this example the vehicle is commanded to zero from an initial roll of $-10 \mathrm{deg}$. Initially the vehicle response lies in the firing region below the switching curves corresponding to a positive roll torque command. As the vehicle rate builds and the position error decreases the vehicle follows the predicted parabolic path in the phase plane until the trajectory intersects the lower switching curve at location \#1. The trajectory then enters the coast region between the lower and upper switching curves where the RCS command turns the roll jets off. Since the rate error is positive, the position error goes toward zero as the trajectory proceeds at constant rate from location $\# 1$ to $\# 2$. At this point the trajectory crosses

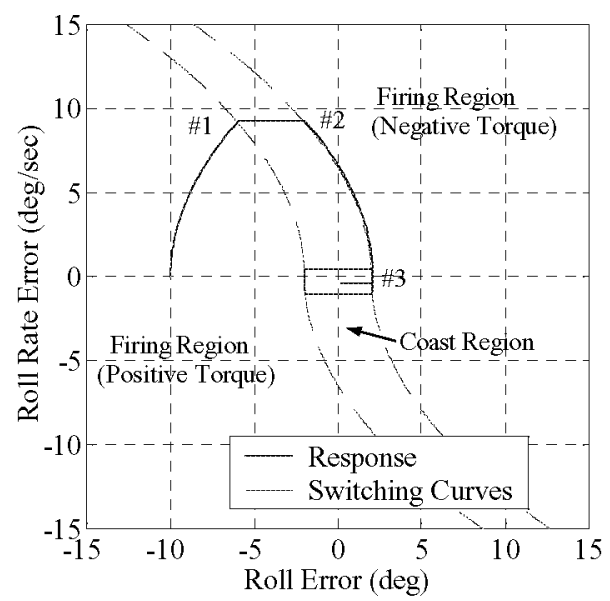

Figure 4 - Roll Phase Plane, Initial Condition Response the upper switching curve and enters the firing region corresponding to negative RCS roll torque. Since the switching curves are defined as the $2^{\text {nd }}$ integral of roll acceleration, i.e. parabolic as described above in Equa-
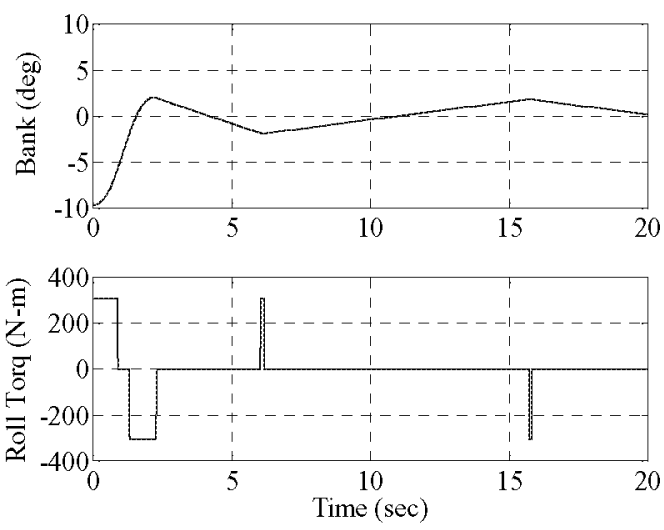

Figure 5-Roll Initial Condition Response

tion (1), the trajectory within the phase plane follows the switching curve to the desired location \#3. At this point the roll response enters a limit cycle and thereafter remains within the roll deadband specification. This example demonstrates how the LEM type phase plane controller can accomplish the task of driving the vehicle response within the desired deadband in reasonable time with minimal overshoot. This maneuver is complete and the vehicle response enters the desired limit cycle with a single bang-bang torque doublet. The resulting optimal response is a trade between fuel consumption and maneuver time for a given RCS angular acceleration. $^{3}$

The roll channel initial conditions responses in Figures 4 and 5 demonstrate the performance of the LEM type phase plane controller in the case of a constant "set-point" command. Consider the performance of this controller in response to a constantly changing "tracking" command such as that from a realistic guidance command sequence. Figure 6 shows the phase plane response and Figure 7 shows the time domain response to a continuously varying "chirp type" roll angle command. The roll angle follows the command reversals with minimal lag and acceptable overshoot at the end of the command sequence. However, the roll torque plot shows that the controller exhibits a high frequency on/off cycling of the RCS jets known as "chattering". Since the command is varying, the dynamics of the error states within the phase plane no longer maintain the predicted parabolic shape during periods when the RCS jets are on. The resulting trajec- 
tories cross back and forth over the switching curves leading to the chattering response. This behavior is undesirable since it may lead to cyclic fatigue of the jet mechanisms and excessive fuel consumption. Since the command dynamics are not known apriori, it is not possible to predict, in general, the error dynamics within the phase plane. Thus one cannot choose an appropriate shape for the switching curves to arrive at the optimal bang-bang response.

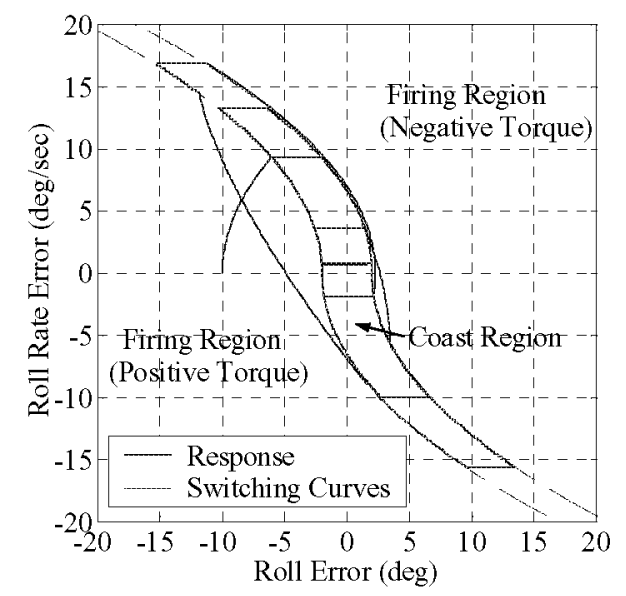

Figure 6-Roll Phase Plane, "Chirp" Cmd. Response
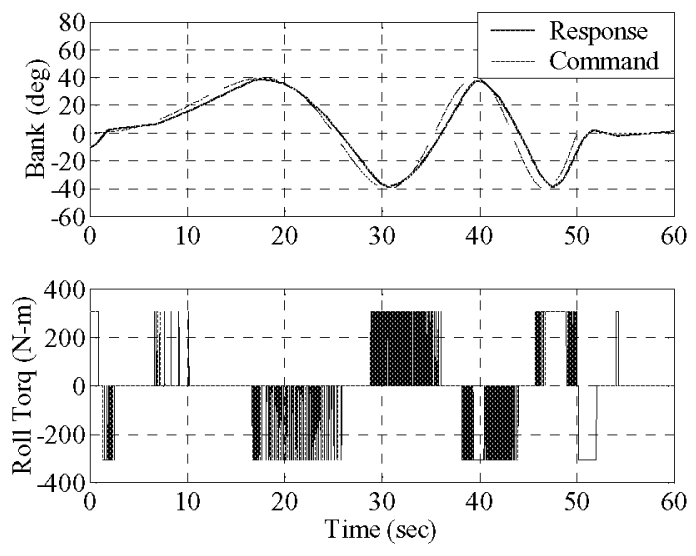

Figure 7 - Roll, “Chirp” Cmd. Response

This issue was addressed in the LEM controller by holding the RCS jets on if the angle error state was being driven to within the deadband at zero rate error while the state trajectory was inside the coast region (i.e., between the switching curves). ${ }^{4}$ To provide a design-flexible means of driving the state toward the de- sired deadband limit cycle, a slight modification was considered for coast region logic. This involved inclusion of an additional set of switching curves to provide hysteresis in the on/off switching characteristics within the coast region. These additional curves were designed to provide good closed loop performance while minimizing the "chattering" behavior of the RCS. A candidate set of "hysteresis switching curves" is shown in the phase plane plot, Figure 10, demonstrating the improved controller behavior. As the trajectory crosses the original switching curves from the coast region to the firing region the RCS jets are commanded on. If the phase plane trajectory enters the region between the original switching curve and the adjacent hysteresis switching curve the RCS jets are commanded to remain on until the trajectory crosses the corresponding hysteresis curve. This allows the jets to remain on for some time after the trajectory crosses the original switching curve, and thus reduces high frequency jet cycling while steering the trajectory towards the desired limit cycle. The width of the hysteresis region was reduced to zero by an amount proportional to the rate error as the rate error became lower than a threshold of about 7 $\mathrm{deg} / \mathrm{sec}$. This shaping of the hysteresis region was used to drive the phase plane trajectory toward the desired location at the termination of the switching curves at zero rate error. The shape of the hysteresis regions are somewhat arbitrary and the candidate switching curves definitions were chosen to provide good command tracking while reducing chattering and fuel usage for MSL conditions.

In addition to the hysteresis switching curves, the phase plane controller was modified by addition of a small delta in the switching curve termination location, as they approach zero rate error from the positive or negative directions. This forces the trajectory to enter the coast region as the rate error changes sign which results in a minimum impulse jet firing limit cycle for periods of constant commands. Constant bank commands are typical guidance outputs at the beginning and end of the entry phase. Similar switching curve termination characteristics where utilized in the LEM controller to achieve minimum impulse firing limit cycle. $^{4}$

\section{$\underline{\text { Simulation Study }}$}

The modified LEM phase plane controller was used for the roll channel of the MSL entry RCS controller and the system performance was tested in a $6 \mathrm{DOF}$ simulation. The simulated responses shown start just after maximum dynamic pressure and continue to about Mach 3. The "chirp-type" command sequence, shown previously, is shown to demonstrate the control system 
performance. A phase plane controller utilizing linear switching curves was used for the angle-of-attack and sideslip channels since large aerodynamic angle maneuvers are not expected, i.e. the vehicle will be commanded to slowly varying pitch trim conditions with zero sideslip command during the entire entry. Figure 8 shows the vehicle angle-of-attack, sideslip and bank angle responses. The aerodynamics exhibit minimal damping in pitch and yaw, hence the angle-of-attack and sideslip oscillatory response about the aerodynamic trim conditions. The bank angle response follows the guidance command with good performance indicated by minimal command response lag and overshoot. Figure 9 shows the RCS torque command demonstrating significant reduction in the high frequency "chattering". Figure 10 shows the phase plane plot for the 6DOF case study along with the complete set of switching curves for the modified controller.

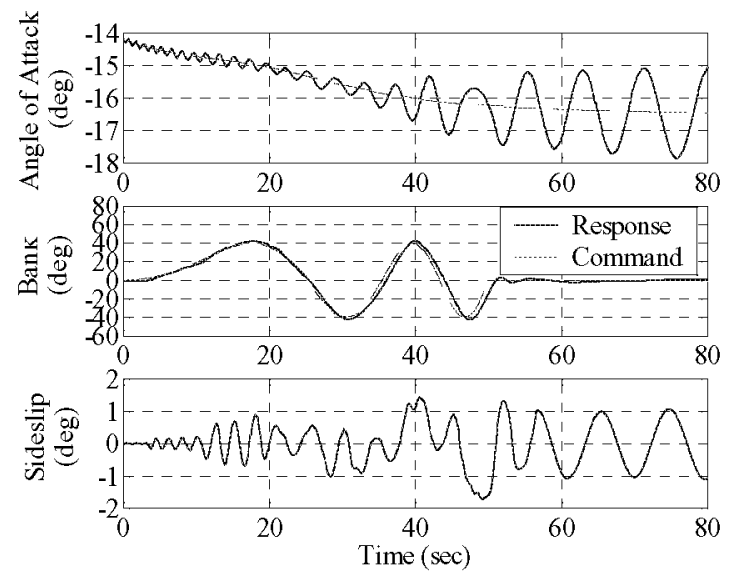

Figure 8-6DOF Case Study, "Chirp” Cmd. Response

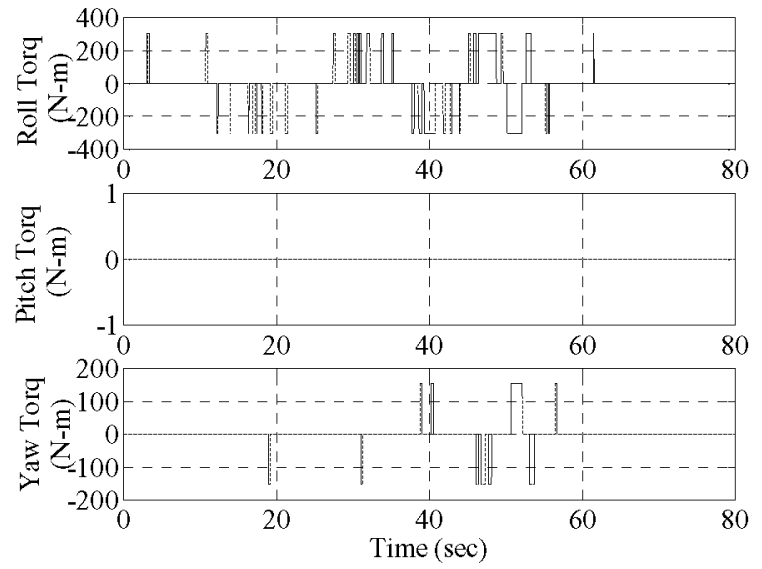

Figure 9-6DOF Case Study, "Chirp" Cmd. RCS Torque

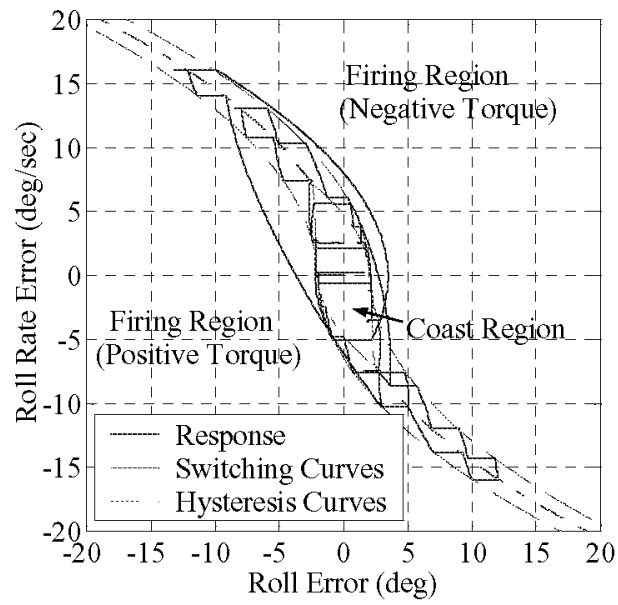

Figure 10-6DOF Case Study, Roll Phase Plane

\section{Concluding Remarks}

A phase-plane controller based on the RCS controller used for the LEM digital autopilot, was used as an entry controller for a Martian surface probe. Since the phase-plane controller is a single-input, single-output system, it is intended for use only on systems without cross-coupling. Aerodynamic cross-coupling exists for the Martian surface probe, but for the levels of coupling anticipated the phase-plane controller is seen to be robust enough to maintain satisfactory performance. A useful controller modification for this application is the inclusion of a set of hysteresis switching curves as an alternate approach for preventing RCS chattering during large angle continuous bank maneuvers. The controller performance was demonstrated in a 6DOF simulation of the candidate entry vehicle configuration.

\section{$\underline{\text { References }}$}

1. Miller, J.E., et al. "Space Navigation Guidance and Control", Technivision Limited, Maidenhead England, August 1966.

2. Brauer, G.L, et al., Program to Optimize Simulated Trajectories (POST), NASA Contract NAS118147, September 1989.

3. Cherry, G.W., Design Principles for an Integrated Guidance \& Control System For the Lunar Excursion Module, AIAA Fourth Manned Space Flight Meeting, St. Louis, Mo, October 11-13 1965.

4. Widnall, W.S., The Lunar Module Digital Autopilot, AIAA Paper No. 70-991, AIAA Guidance, 
Control and Flight Mechanics Conference, Santa Barbara, Ca. , August 17-19 1970.

5. Justus, C.G., James, B.F., Mars Global Reference Atmosphere Model 2000 version (Mars-GRAM 2000): User's Guide, NASA TM-2000-210279, May 2000.

6. "Using Simulink", The Mathworks, 1996.

7. "Matlab Reference Guide", The Mathworks, 1993.

8. Bobskill, G.J., Parikh, P.C., Prabhu, R. K., Tyler, E.D., Aerodynamic Database Development for Mars Smart Lander Vehicle Configurations, AIAA Paper No. 2002-4411, AIAA Atmospheric Flight Mechanics Conference, Monterey, Ca., Aug 5-8 2002. 